\title{
Nonequilibrium chiral perturbation theory and Disoriented Chiral Condensates $^{1}$
}

\author{
Angel Gómez Nicola ${ }^{2}$ \\ Departamento de Física Teórica \\ Universidad Complutense, 28040, Madrid, Spain
}

\begin{abstract}
We analyse the extension of Chiral Perturbation Theory to describe a meson gas out of thermal equilibrium. For that purpose, we let the pion decay constant be a time-dependent function and work within the Schwinger-Keldysh contour technique. A useful connection with curved space-time QFT allows to consistently renormalise the model, introducing two new low-energy constants in the chiral limit. We discuss the applicability of our approach within a Relativistic Heavy-Ion Collision environment. In particular, we investigate the formation of Disoriented Chiral Condensate domains in this model, via the parametric resonance mechanism.
\end{abstract}

\section{INTRODUCTION AND MOTIVATION}

The forthcoming experiments on Relativistic Heavy Ion Collision (RHIC) at BNL and CERN will be able to test accurately the dynamics of the QCD plasma. After the collision, the plasma formed in the central rapidity region cools down via hydrodynamic expansion, and nonequilibrium effects become important in that regime. Among them, one of the most interesting suggestions has been the formation of the so called Disoriented Chiral Condensates (DCC). The DCC were proposed originally in [1] as misaligned vacuum regions, where the chiral field points out in directions in isospin space different from that where the vacuum expectation value of the pion field vanishes. If such regions were formed, one could observe large clusters of pions emitted coherently from the plasma as the pion field relaxes to the normal vacuum. This kind of behaviour is indeed observed in Centauro and anti-Centauro events in cosmic ray experiments [2]. However, one should point out that a clear signal for DCC formation has not been observed yet in the RHIC at Fermilab [3], although it could well happen that the DCC's are too small to be directly detected and one has to think of alternative observables (see below).

1) Talk given in "Hadron Physics: Effective Theories of Low Energy QCD", Coimbra, Portugal.

2) E-mail: gomez@eucmax.sim.ucm.es 
On the other hand, after the hadronisation time, a proper description of the microscopic meson dynamics makes it compulsory to use an effective low-energy theory for QCD. In this context, the chiral symmetry plays a fundamental role. The effective theory must incorporate all the QCD symmetries and the chiral spontaneous symmetry breaking (SSB) pattern, so that the Nambu-Goldstone bosons (NGB) are the lightest mesons $(\pi, K, \eta)$. The light quark masses are then introduced perturbatively. One possible choice is simply an $O(N)$ model, with the standard classical SSB potential. Its fundamental fields are the $N-1$ pions and the $\sigma$, which acquires a nonzero vacuum expectation value $v$. This is the Linear Sigma Model (LSM) description. However, one should bear in mind that the LSM becomes nonperturbative in the coupling constant at low energies, so that alternative perturbative expansions have to be used, such as large $N$. Besides, the LSM only shares the QCD chiral symmetry breaking pattern for $N=4$. An alternative approach is to construct an effective theory as an infinite sum of terms with increasing number of derivatives, only for the NGB fields. This is the description based in the Nonlinear Sigma Model (NLSM), which is the lowest order action one can write down in this expansion. Higher order corrections come both from NGB loops and higher order lagrangians and can be renormalised order by order in energies, yielding finite predictions for the meson observables. The unknown coefficients, which encode all the information on the underlying theory, absorb the loop infinities and can be fitted to experiment. This framework constitutes the so called chiral perturbation theory (ChPT) $[4,5]$. The perturbative expansion is carried out in terms of the ratio of the $\mathcal{O}(p)$ meson energy scales of the theory (masses, external momenta, temperature and so on) and the chiral scale $\Lambda_{\chi} \simeq 1 \mathrm{GeV}$ (see [6-9] for a review).

Nonequilibrium effects such as the DCC's have been investigated in the literature using $O(N)$ models with initial thermal equilibrium conditions $\sigma(t=0)=0$ and $\pi^{a}(t=0)=0$. In this context, two different scenarios for DCC formation have been proposed: the first one takes place in the early stages of the plasma evolution. Roughly speaking, as the field rolls down along the potential, long wavelength modes grow exponentially and enhance the formation of DCC's. There have been several approaches in the literature to implement this idea in the $O(N)$ model, like classical simulations [10], large $N$ [11], or analysis based on reasonable assumptions on the kinematics $[12,13]$. Typical DCC sizes within this approach are of the order of $2-3 \mathrm{fm}$, containing $n_{\pi} \simeq 0.2 \mathrm{fm}^{3}$ pions, whereas the plasma cools down in a proper time of about $\tau \simeq 5-10 \mathrm{fm} / \mathrm{c}$. As commented above, these numbers yield too small DCC's to be observed directly. A second suggestion, which has been recently proposed [14-16] is based on the parametric resonance mechanism and inherits the idea from inflationary reheating [17]. In this approach, the $\sigma$ field is very close to the bottom of the potential but it is still oscillating around it (it clearly overshoots the vacuum if the initial conditions are imposed on the top) in the late stage of the plasma evolution. Those oscillations transfer energy to the pion modes, giving rise to exponentially growing pion solutions for certain bands in momentum space, via parametric resonance. Recent work within the LSM in this approach predicts rather larger DCC, of sizes up to $5 \mathrm{fm}$ [15]. More details about 
this mechanism will be given below.

In the present work, we will explore the applicability of ChPT to describe the meson plasma out of thermal equilibrium. So far, this formalism has been applied only in equilibrium, to study the low $T(T=\mathcal{O}(p))$ meson gas and the chiral phase transition $[18,19]$. The key idea of our approach is to make use of the derivative expansion consistently defined in ChPT in order to study the system not far from equilibrium. It is therefore best suited for the late stage evolution and has the additional advantages typical of standard ChPT, i.e, it deals only with NGB fields and is equally applicable to three flavours. We will show that a systematic power counting can be established in this case and, furthermore, that the renormalisation program can be consistently implemented. Details can be found in [20]. In addition, in the last section we will explore the possibility of describing DCC formation within ChPT, via parametric resonance.

\section{THE MODEL AND CHIRAL POWER COUNTING}

Our starting point is the nonlinear sigma model (NLSM) where we let the pion decay constant- the only relevant parameter to the lowest order in derivatives- be time dependent. In the context of a RHIC, such time dependence can be thought of as proper time evolution within the so called Bjorken initial conditions [21], where observables depend only on proper time and not on rapidity. This picture is consistent with the experimental observations. We take the initial time $t=0$, having in mind that it would correspond to a proper time $\tau_{0} \simeq 1-2 \mathrm{fm} / \mathrm{c}$, a typical hadronisation time. Thus, we will consider the following NLSM action

$$
S[U]=\int_{C} d t \int d^{3} \vec{x} \frac{f^{2}(t)}{4} \operatorname{tr} \partial_{\mu} U^{\dagger}(\vec{x}, t) \partial^{\mu} U(\vec{x}, t)
$$

Here, $C$ is the Schwinger-Keldysh contour (see [20] for details), which parametrises the nonequilibrium path integral where we are considering thermal equilibrium for $t \leq 0$ at a temperature $T_{i}=\beta_{i}^{-1}$, as the initial condition. Note that the action $(1)$ is chiral invariant $\left(U \rightarrow L U R^{\dagger}\right)$ by construction, which will play an important role in what follows. As a first approximation, we will be interested only in the strict chiral limit for two light flavours, i.e, massless pions. Therefore, we are not including any explicit symmetry-breaking term in the action. Thus, $f(t \leq 0)=f \simeq 93 \mathrm{MeV}$ to leading order $\left(f \neq f_{\pi}\right.$ to higher orders $)$ and for $t>0$ the system departs from equilibrium. Note that, since we choose that departure to be instantaneous, $f(t)$ cannot be analytical at $t=0$. This is just an artifact of the approximation and should not have any effect on the long-time behaviour. Finally, as customary, $U(x)$ is parametrised in terms of pion fields $\pi^{a}$ as:

$$
U(\vec{x}, t)=\frac{1}{f(t)}\left\{\left[f^{2}(t)-\pi^{2}\right]^{1 / 2} I+i \tau_{a} \pi^{a}\right\}
$$

and $\pi^{a}\left(t_{i}-i \beta_{i}\right)=\pi^{a}\left(t_{i}\right)$ is the equilibrium boundary condition, with $t_{i}<0$. 
The new ingredient we need to incorporate in the power counting in order to be consistent with ChPT is then

$$
\frac{\dot{f}(t)}{f^{2}(t)} \simeq \mathcal{O}\left(\frac{p}{\Lambda_{\chi}}\right), \quad \frac{\ddot{f}(t)}{f^{3}(t)}, \frac{[\dot{f}(t)]^{2}}{f^{4}(t)} \simeq \mathcal{O}\left(\frac{p^{2}}{\Lambda_{\chi}^{2}}\right),
$$

and so on. Obviously, our results will depend upon the choice of $f(t)$. One can think of $f(t)$ as an external source, to which we wish to obtain the nonequilibrium response of the system. Alternatively, this model can be thought of to lowest order as the LSM with the time-dependent constraint $\sigma^{2}+\pi^{2}=f^{2}(t)$. We shall discuss below a reasonable assumption for $f(t)$ in connection with DCC formation. Meanwhile, we shall keep $f(t)$ arbitrary.

To lowest order in the pion fields, the above NLSM action can be written as

$$
S_{0}[\pi]=-\frac{1}{2} \int_{C} d^{4} x \pi^{a}(\vec{x}, t)\left[\square+m^{2}(t)\right] \pi^{a}(\vec{x}, t)
$$

where $\int_{C} d^{4} x=\int_{C} d t \int d^{3} \vec{x}$ and $m^{2}(t)=-\ddot{f}(t) / f(t)$. That is, the model accommodates a time-dependent pion mass term, without breaking explicitly the chiral symmetry. This effect is the same as switching on an external curved space-time background, as we will see in the next section.

\section{RENORMALISATION AND CURVED SPACE-TIME}

Once we have defined our nonequilibrium power counting, we can apply ChPT to calculate the time evolution of the observables. In doing so, we must pay special attention to renormalisation. The fact that there is a time-dependent mass term indicates that there can be new time-dependent infinities in the chiral loops. However, we are in the chiral limit, so we are not allowed to introduce the usual $\mathcal{O}\left(p^{4}\right)$ mass and wave function counterterms breaking the chiral symmetry [5]. In other words, we should be able to construct the most general fourth order action, which in particular should include new terms (and hence new low-energy constants) to cancel those extra divergences, preserving exactly the chiral symmetry.

In order to find this $\mathcal{O}\left(p^{4}\right)$ lagrangian, we will make use of a very fruitful analogy: the action (1) is equivalent to formulate the NLSM on a curved space-time background corresponding to a spatially flat Robertson-Walker metric, with scale factor $a(t)=f(t) / f\left(0^{+}\right)$(see [20] for details). Note that in this language, $m^{2}(t)$ in $(4)$ represents the minimal coupling with the RW metric preserving chiral invariance.

Therefore, we can construct the $\mathcal{O}\left(p^{4}\right)$ action as:

$$
S_{4}[U, g, R]=\int_{C} d^{4} x \sqrt{-g}\left[\mathcal{L}_{4}[U, g]+\left(L_{11} R g^{\mu \nu}+L_{12} R^{\mu \nu}\right) \operatorname{tr} \partial_{\mu} U^{\dagger} \partial_{\nu} U\right]
$$

where $g$ is the metric determinant, $\mathcal{L}_{4}[U, g]$ stands for the standard (equilibrium) lagrangian [5] with indices raised and lowered with the $g^{\mu \nu}$ metric and the rest are 
new $\mathcal{O}\left(p^{4}\right)$ invariant couplings with the scalar curvature $R(x)$ and the Ricci tensor $R_{\mu \nu}(x)$ in the chiral limit. These are the new terms we need, where $L_{11}$ and $L_{12}$ are the new coupling constants. In fact, this problem has been already considered in [22] in order to study the energy-momentum tensor of QCD at low energies. In that work it has been found that $L_{11}$ is renormalised in dimensional regularisation, whereas $L_{12}$ is already finite. Their numerical values can be obtained from the experimental information on the QCD energy-momentum form factors. They yield $L_{12} \simeq-2.7 \times 10^{-3}$ and $L_{11}^{r}(\mu=1 G e V) \simeq 1.4 \times 10^{-3}$ where $\mu$ is the renormalisation scale. In our case, with our RW metric we get to $\mathcal{O}\left(\pi^{2}\right)$,

$$
S_{4}[\pi, g]=-\frac{1}{2} \int_{C} d^{4} x \pi^{a}\left[f_{1}(t) \partial_{t}^{2}-f_{2}(t) \nabla^{2}+m_{1}^{2}(t)\right] \pi^{a}+\mathcal{O}\left(\pi^{4}\right)
$$

with

$$
\begin{aligned}
& f_{1}(t)=12\left[\left(2 L_{11}+L_{12}\right) \frac{\ddot{f}(t)}{f^{3}(t)}-L_{12} \frac{[\dot{f}(t)]^{2}}{f^{4}(t)}\right] \\
& f_{2}(t)=4\left[\left(6 L_{11}+L_{12}\right) \frac{\ddot{f}(t)}{f^{3}(t)}+L_{12} \frac{[\dot{f}(t)]^{2}}{f^{4}(t)}\right] \\
& m_{1}^{2}(t)=-\left[\frac{f_{1}(t) \ddot{f}(t)+\dot{f}_{1}(t) \dot{f}(t)}{f(t)}+\frac{1}{2} \ddot{f}_{1}(t)\right]
\end{aligned}
$$

The above lagrangian should take care of the nonequilibrium infinities we might find in the pion two-point function. We will see below that this is indeed the case.

\section{THE PION DECAY FUNCTIONS $f_{\pi}(T)$}

The first observable one might think of calculating in ChPT is the pion decay constant to one loop. In the nonequilibrium model, it will become a time-dependent function $f_{\pi}(t)$. One should point out that the definition of $f_{\pi}$ is subtle even in thermal equilibrium $[19,23]$. In addition, one has in general $f_{\pi}^{s}(T) \neq f_{\pi}^{t}(T)$ corresponding to the axial current spatial and temporal components and due to the loss of Lorentz covariance in the thermal bath [24]. We refer to [20] for details on how to define properly $f_{\pi}(t)$ out of equilibrium. Once this has been done, one has to consider the one loop diagrams for the pion two-point function coming from (1) plus the tree level ones from (6). The final result up to $\mathcal{O}\left(p^{4}\right)$ reads [20]

$$
\begin{aligned}
{\left[f_{\pi}^{s}(t)\right]^{2} } & =f^{2}(t)\left[1+2 f_{2}(t)-f_{1}(t)\right]-2 i G_{0}(t) \\
{\left[f_{\pi}^{t}(t)\right]^{2} } & =f^{2}(t)\left[1+f_{2}(t)\right]-2 i G_{0}(t)
\end{aligned}
$$

for $t>0$, with $f_{1,2}(t)$ in $(7)$ and $G_{0}(t)$ is nothing but the equal-time pion two-point function $G_{0}(t)=G_{0}(x, x)$ with $G_{0}(x, y)$ the solution of the differential equation

$$
\left\{\square_{x}+m^{2}\left(x^{0}\right)\right\} G_{0}(x, y)=-\delta_{C}\left(x^{0}-y^{0}\right) \delta^{(3)}(\vec{x}-\vec{y})
$$


with KMS equilibrium conditions $G_{0}^{>}\left(\vec{x}, t_{i}-i \beta_{i} ; y\right)=G_{0}^{<}\left(\vec{x}, t_{i} ; y\right), G_{0}^{>}$and $G_{0}^{<}$ being advanced and retarded correlation functions. Clearly, this equation cannot be solved analytically for an arbitrary $f(t)$, but it can be managed numerically. Therefore, one must remember that $G_{0}(t)$ depends implicitly on $f(t)$ through $(10)$.

As a consistency check, the results (8)-(9) reproduce the equilibrium result [25] when we switch off the time derivatives of $f(t)$ :

$$
\left[f_{\pi}^{s}(T)\right]^{2}=\left[f_{\pi}^{t}(T)\right]^{2}=f^{2}\left(1-\frac{T^{2}}{6 f_{\pi}^{2}}\right)
$$

An interesting consequence of our result is that $f_{\pi}^{s}(t) \neq f_{\pi}^{t}(t)$ to one-loop, unlike the equilibrium case. In addition, from (8)-(9) and (7) we see that the difference $\left[f_{\pi}^{s}(t)\right]^{2}-\left[f_{\pi}^{t}(t)\right]^{2}$ is finite, so that $f_{\pi}^{s}(t)$ and $f_{\pi}^{t}(t)$ can be renormalised at the same time, which is another consistency check. We remark that $G_{0}(t)$ contains in general UV divergences, to be absorbed by $f_{1}(t)$ and $f_{2}(t)$ in the renormalisation of $L_{11}$. An explicit check of this renormalisation procedure will follow in the next section.

\section{DISORIENTED CHIRAL CONDENSATES IN CHPT}

In this section we will consider a particular choice of $f(t)$ and apply our previous results. Our motivation is the possibility of generating DCC-like structures in this context. We shall sketch some of our preliminary results here, while details of the calculation and further work will be postponed to a forthcoming paper.

As we have discussed above, our approach is meant to be useful in a stage of the plasma evolution where the departure from equilibrium is of the same order as the meson energies. Hence, we should be able to obtain similar results as the analysis performed in the LSM in the parametric resonance regime [14-16], where the rolling down of the $\sigma$ field is in its late oscillatory period. This is the same behaviour of the inflaton field in reheating [17]. One then allows for a time-dependent classical background $\sigma(t)$ in the LSM, splitting the field as

$$
\sigma(\vec{x}, t)=\sigma(t)+\delta \sigma(\vec{x}, t)
$$

where $\delta \sigma$ is the quantum fluctuation. As a first approximation, one can neglect the pion fluctuations $\left\langle\pi^{2}\right\rangle \ll v^{2}[14,16]$ and solve the equation of motion, which yields just $\sigma(t)=\sigma_{0} \cos m_{\sigma} t$. Here, $\sigma_{0}$ is the initial field amplitude, which in this approximation is a small quantity. Even though, one can still produce exponentially growing pion fields (DCC) which in the end will be responsible for the damping of the oscillations as the field relaxes to equilibrium. One should bear in mind that neglecting $\left\langle\pi^{2}\right\rangle$ to lowest order is a rather crude approximation, as pointed out in [15], which is clearly not valid for large times when the pion correlator grows significantly. Nonetheless, we will carry on with this simple case, just to understand qualitatively how ChPT can also account for the description of DCC's. A better approximation would be to solve the coupled equations for the $\sigma$ and $\pi$ fields, 
which yields the solution for $\sigma(t)$ in terms of elliptic functions [15]. Therefore, in this simple picture, we take our $f(t)$ of the same form as the lowest order $\sigma(t)$ in the LSM, i.e,

$$
f(t)=f\left[1-\frac{q}{2}(\cos M t-1)\right]
$$

Here, $q$ is a small parameter, playing the role of $\sigma_{0}$ in the LSM. Notice that our nonequilibrium chiral power counting demands $q M^{2}=\mathcal{O}\left(p^{2}\right)$ and so on. Thus, for definiteness, we will take $q=\mathcal{O}\left(p^{2} / \Lambda_{\chi}^{2}\right)$, so that the $\mathcal{O}\left(p^{4}\right)$ corrections remain under control (see below), and $M$ arbitrary. In the end, we will discuss how the results are affected by $T_{i}, q$ and $M$. Therefore, we have $m^{2}(t)=-\left(q M^{2} / 2\right) \cos M t(1+\mathcal{O}(q))$, so that the differential equation (10) becomes to leading order

$$
\left[\frac{d^{2}}{d t^{2}}+\frac{4 k^{2}}{M^{2}}-2 q \cos M t\right] G_{0}^{>}\left(k, t, t^{\prime}\right)=0
$$

where we have Fourier transformed in the spatial coordinates only $\left(k^{2}=|\vec{k}|^{2}\right)$. The above equation is nothing but the Mathieu equation, which has several wellknown interesting properties $[26,27]$. Among them, it admits unstable solutions exponentially growing in time, for certain values of $4 k^{2} / M^{2}$. This is the simplest version of the parametric resonance mechanism. In particular, the instabilities develop in bands in $k$, centered at $k_{n}=n M / 2$, of width $\Delta k_{n}=\mathcal{O}\left(q^{n}\right)$. Hence, in the

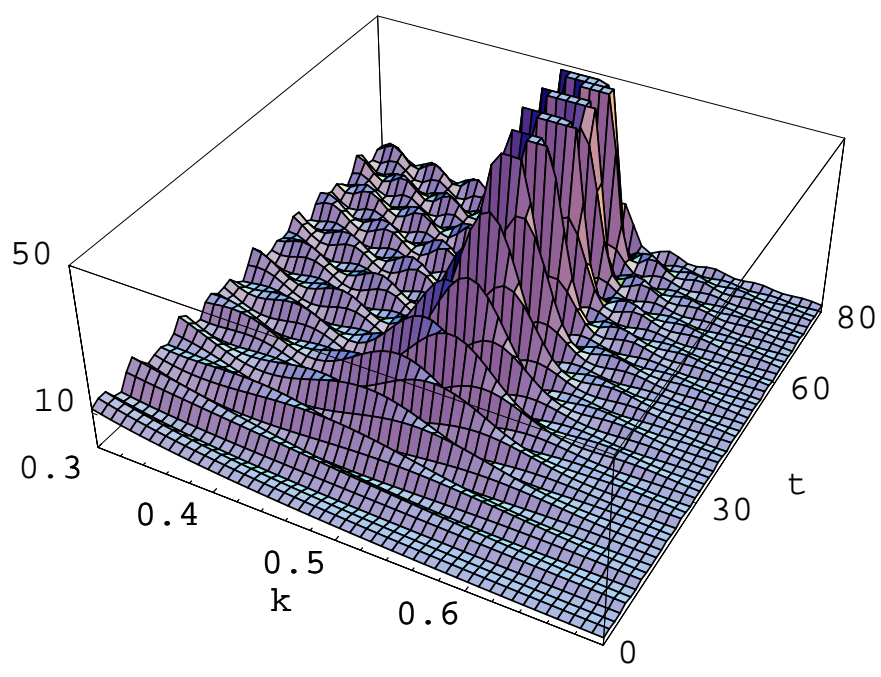

FIGURE 1. $i M G_{0}(k, t)$ for $T_{i}=M$ and $q=0.1$. Spatial momentum and time are measured in units of $M$ and $M^{-1}$ respectively. The instability band for this case lies roughly between $0.4 M<k<0.6 M$. 
approximation we are working, only the first band is relevant, i.e, unstable solutions only exist for $M / 2-\Delta k_{1}<k<M / 2+\Delta k_{1}$. This is known in the Cosmology literature as the narrow resonance approximation [17]. A typical unstable solution $G_{0}(k, t)$ has been plotted in Figure 1 for a particular choice of the parameters in the first band. The solutions typically oscillate with an exponentially growing amplitude inside the unstable region. Therefore, we see that our ChPT approach allows for DCC-type configurations.

Next, we will apply our results for $f_{\pi}(t)$ to this particular case. The equaltime correlation function $G_{0}(t)=\int d^{3} k G_{0}(k, t, t)$ turns out to be UV divergent, as expected. After standard manipulations in dimensional regularisation $(d=4-\epsilon)$ one can cast the divergent part for $t>0$ as

$$
i G_{0}^{d i v}(t)=-\frac{q M^{2}}{16 \pi^{2}} \cos M t\left(\frac{1}{\epsilon}+\frac{1}{2} \log \frac{\mu^{2}}{M^{2}}\right)
$$

which is an example of the new time-dependent divergences we were talking about in previous sections. In fact, we see that it has exactly the same form as $\ddot{f}(t) / f(t)$. Furthermore, replacing in (8)-(9), we find that the result is rendered finite and scale independent with the same renormalisation of $L_{11}$ derived in [22].

The final results for $f_{\pi}^{s}(t)$ are plotted in Figure 2 for different choices of the parameters. We clearly observe the damping effect on the amplitude due to the unstable solutions at long times. In other words, the DCC's accelerate thermalisation. We also observe that this mechanism becomes less efficient for smaller $q$ and $M$. Typically, the unstable corrections to the amplitude of $f_{\pi}(t)$ are proportional to $\left(q M^{2} / 4 \pi f_{\pi}^{2}\right) \exp (q M t)$. On the other hand, this effect seems to be rather insensitive to the initial temperature and thus we expect to catch all the important qualitative behaviour concerning the DCC's, regardless of the initial conditions. It should be pointed out that the curves have been cut off at the times where the one-loop contribution becomes of the same size as the tree level one. From that point onwards, the exponentially growing correlator dominates, yielding unphysical results. As commented above, we do not expect our simple cosine shape for $f(t)$ to be valid for all times, since it is derived neglecting the pion correlator. This final time $t_{f}$ roughly defines the applicability range of our results. We expect that this range is enough to account for all the plasma time evolution of a realistic RHIC. For instance, for $M=1 \mathrm{GeV}$ and $q=0.1$, we get $t_{f} \simeq 35 M^{-1} \simeq 8 \mathrm{fm} / \mathrm{c}$. This is exactly the same as extrapolating the equilibrium result (11) to predict the critical temperature at $T=T_{c} \simeq 6 f_{\pi}^{2}$, where all the higher order corrections become of the same order. Nonetheless, that formula predicts the right behaviour of $f_{\pi}(T)$ as it approaches the transition. In the same way, our results reproduce the expected qualitative behaviour as we extrapolate them up to times $t \simeq t_{f}$. Therefore, $f_{\pi}(t)$ can be regarded as an alternative observable (it is the residue of an axial-axial correlator and it can be measured in semileptonic decays) to test the size of DCC-like configurations in the late stage of the plasma expansion. 

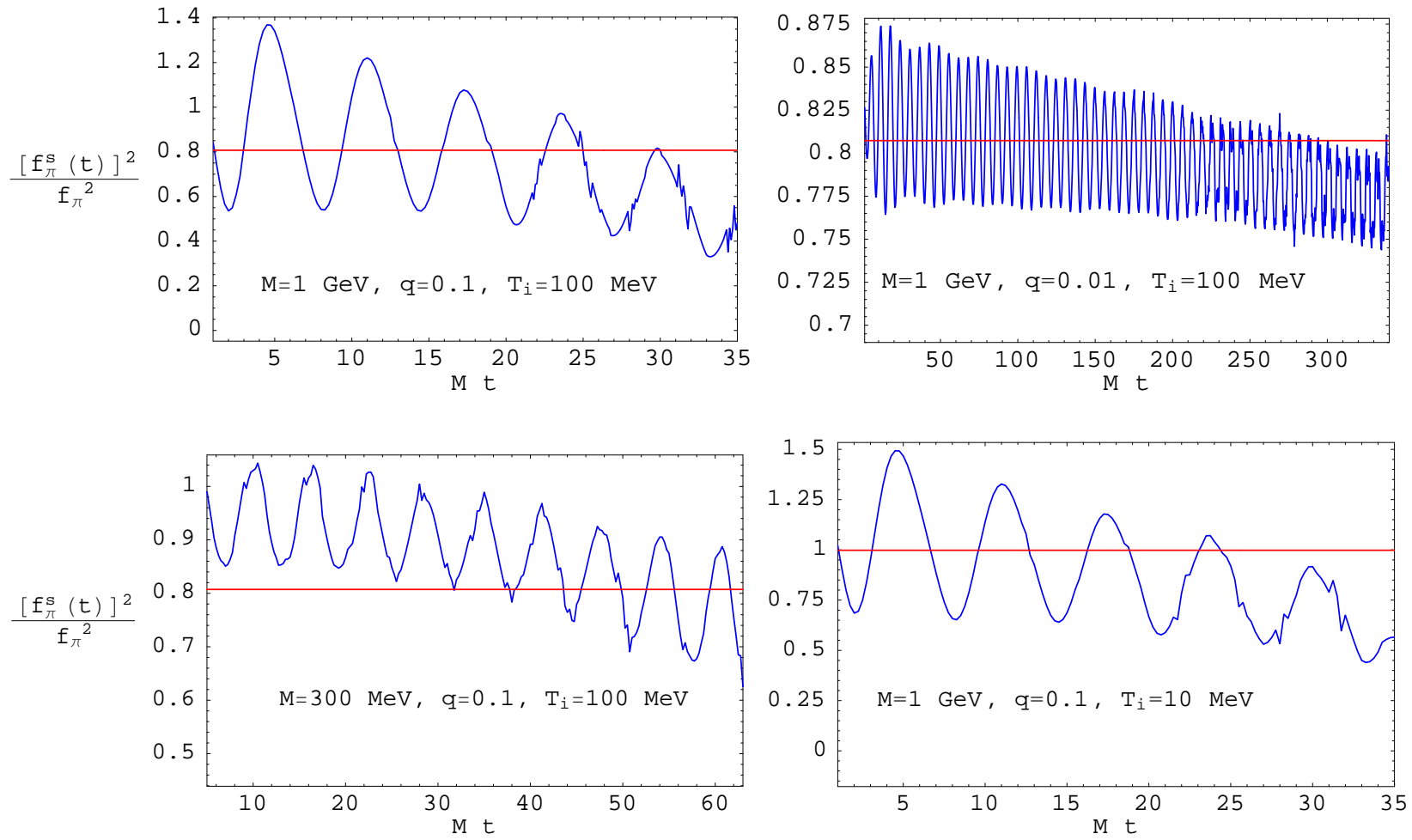

FIGURE 2. Results for $f_{\pi}^{s}(t)$ in the parametric resonance regime. The horizontal line is the value of $f_{\pi}$ at the initial temperature $T_{i}$

\section{CONCLUSIONS AND OUTLOOK}

We have reviewed recent work on the extension of ChPT to a nonequilibrium situation. The NLSM with a time dependent pion decay constant provides a nonequilibrium effective model with a well-defined perturbative expansion and power counting near equilibrium. The analogy of this model with curved space-time QFT allows to consistently construct higher order lagrangians and implement renormalisation. As a first application, we have obtained the renormalised one-loop $f_{\pi}(t)$.

We have also shown how this model can be applied to describe DCC-like structures in the late stage of the expansion of a hot plasma formed after a RHIC. Work in progress includes a more realistic study of the parametric resonances, including consistently the pion correlations in $f(t)$ and calculating the correlation length and the number of pions. One can also think of including pion masses, extending the results to three flavours, using large $N$ methods and cosmological applications as other interesting aspects to be investigated in this context. 


\section{ACKNOWLEDGMENTS}

I wish to thank the organisers of the "Hadron Physics" conference and the Theory group in Coimbra for their kind help and hospitality. Financial support from CICYT, Spain, project AEN97-1693, is also acknowledged.

\section{REFERENCES}

1. A.Anselm, Phys. Lett. B217 (1989) 169; A.Anselm and M.Ryskin, Phys. Lett. B226 (1991) 482 J.D.Bjorken, Int. J. Mod. Phys. A7 (1992) 4189; J.P.Blaizot and A.Krzywicki, Phys. Rev. D46 (1992) 246.

2. L.T.Baradzei et al, Nucl. Phys. B370 (1992) 365.

3. T.Brooks et al, Phys. Rev. D55 (1997) 5667.

4. S.Weinberg, Physica A96 (1979) 327.

5. J.Gasser and H.Leutwyler, Ann. Phys. (N.Y.) 158 (1984) 142, Nucl. Phys. B250 (1985) 465.

6. J.F.Donoghue, E.Golowich and B.R.Holstein, Dynamics of the Standard Model, Cambridge University Press 1992.

7. U-G.Meißner, Rep.Prog.Phys. 56, 903-996, 1993.

8. A.Pich, Rep.Prog.Phys. 58, 563-610, 1995.

9. A.Dobado, A.Gómez Nicola, A.López-Maroto and J.R.Peláez, Effective lagrangians for the Standard Model, Springer 1997.

10. K.Rajagopal and F.Wilczek, Nucl. Phys. B404 (1993) 577.

11. D.Boyanowsky, H.J. de Vega and R.Holman, Phys. Rev. D51 (1995) 734.

12. F.Cooper, Y.Kluger, E.Mottola and J.P.Paz, Phys. Rev. D51 (1995) 2377.

13. M.A.Lampert, J.F.Dawson and F.Cooper, Phys. Rev. D54 (1996) 2213.

14. S.Gavin and B.Müller, Phys. Lett. B329 (1994) 486; S.Mrowczynski and B.Müller, Phys. Lett. B363 (1995) 1.

15. D.Kaiser, Phys. Rev. D56 (1997) 706; Phys. Rev. D59, 117901

16. H.Hiro-Oka and H.Minakata, Phys. Lett. B425 (1998) 129; B434 (1998) 461-462 (E).

17. L.Kofman, A.Linde and A.Starobinsky, Phys. Rev. D56 (1997) 3258.

18. P.Gerber and H.Leutwyler, Nucl. Phys. B321 (1989) 387.

19. A.Bochkarev and J.Kapusta, Phys. Rev. D54 (1996) 4066.

20. A.Gómez Nicola and V.Galán-González, Phys. Lett. B449 (1999) 288-298.

21. J.D.Bjorken, Phys. Rev. D27 (1983) 140.

22. J.F.Donoghue and H.Leutwyler, Z. Phys. C52 (1991) 343.

23. J.I.Kapusta and E.V.Shuryak, Phys. Rev. D49 (1994) 4694.

24. R.D.Pisarski and M.Tytgat, Phys. Rev. D54 (1996) R2989.

25. J.Gasser and H.Leutwyler, Phys. Lett. B184 (1987) 83.

26. N.W.Mac Lachlan, Theory and Application of Mathieu Functions, Dover (New York), 1961.

27. M.Abramowitz and I.A.Stegun, Handbook of Mathematical Functions, Dover (New York), 1970. 